\title{
Protocol Treatment Arm
}

National Cancer Institute

\section{Source}

National Cancer Institute. Protocol Treatment Arm. NCI Thesaurus. Code C15538.

A specific treatment plan within a clinical trial that describes the activities a subject will be involved in as he or she progresses through the study. 